GA-A24502

\title{
Characterization of Peeling-Ballooning Stability Limits on the Pedestal
}

\author{
by \\ P.B. Snyder, H.R. Wilson, ${ }^{\star}$ T.H. Osborne and A.W. Leonard
}




\section{DISCLAIMER}

This report was prepared as an account of work sponsored by an agency of the United States Government. Neither the United States Government nor any agency thereof, nor any of their employees, makes any warranty, express or implied, or assumes any legal liability or responsibility for the accuracy, completeness, or usefulness of any information, apparatus, product, or process disclosed, or represents that its use would not infringe privately owned rights. Reference herein to any specific commercial product, process, or service by trade name, trademark, manufacturer, or otherwise, does not necessarily constitute or imply its endorsement, recommendation, or favoring by the United States Government or any agency thereof. The views and opinions of authors expressed herein do not necessarily state or reflect those of the United States Government or any agency thereof. 


\title{
Characterization of Peeling-Ballooning Stability Limits on the Pedestal
}

\author{
by
P.B. Snyder, H.R. Wilson, ${ }^{*}$ T.H. Osborne and A.W. Leonard \\ "EURATOM/UKAEA Fusion Association, Culham Science Centre, Abingdon, Oxon UK
}

This is a preprint of a paper to be submitted for publication in Plasma Physics and Controlled Fusion.

\author{
Work supported by \\ the U.S. Department of Energy under \\ Contract Nos. DE-AC03-99ER54463 \\ and DE-FG03-95ER54309
}

GENERAL ATOMICS PROJECT 30033 and 03726 March 2004 


\begin{abstract}
The peeling-ballooning model for edge localized modes (ELMs) and pedestal constraints, based upon ideal MHD instabilities driven by pressure gradients and current in the edge barrier region, has been broadly applied toward understanding ELM and pedestal behavior in a number of tokamak experiments. Due in part to multiple driving terms, multiple wavelengths and second stability access physics, the peeling-ballooning stability limits which are proposed to constrain the pedestal and trigger ELMs depend sensitively on many details of the tokamak equilibrium. Here we present a technique for characterizing these stability constraints as a function of important parameters, using carefully constructed model equilibria. We discuss comparisons of calculated stability constraints to observed pedestal behavior, in which an encouraging level of agreement is found. We then present results of an extensive series of calculations which characterize the peeling-ballooning stability constraints as a function of pedestal width, magnetic field, plasma current, density, and triangularity.
\end{abstract}




\section{INTRODUCTION AND BACKGROUND}

Understanding the physics which governs the edge transport barrier region (or "pedestal") in high performance ("H-mode") tokamak plasmas is of critical importance to future burning plasma tokamak devices for two primary reasons. The first is the strong dependence of core confinement on the pressure at the top of the pedestal (or "pedestal height"), which has been observed in experiment as well as predicted by a range of transport models. The second is the presence of edge localized modes (ELMs), which are discrete, repetitive magnetic perturbations in the pedestal vicinity which transport bursts of energy and particles from closed to open magnetic field lines and then to the material surfaces of the divertor or vessel wall. While ELMs are generally benign in present experiments, they pose a significant material erosion risk in planned burning plasma devices.

Many theoretical studies of ELMs have focused on MHD instabilities. Early work focuses on infinite toroidal mode number $(\mathrm{n})$ ballooning modes or low $\mathrm{n}$ kinks [1], and recent work incorporates peeling modes and peeling-ballooning mode coupling, initially in the high $\mathrm{n}$ s-alpha limit [2], and more recently with finite-n corrections and realistic geometry, and emphasizing the important role of intermediate-n $(3<\mathrm{n}<40)$ coupled peeling-ballooning modes as commonly the limiting instability in the pedestal [3]. These recent studies develop what is currently referred to as the peeling-ballooning model of ELMs and pedestal constraints. A key element of this model is that the peeling-ballooning modes provide an effective limit on the pedestal height (at a given pedestal width) as well as driving the ELMs. Of course the model must be combined with an understanding of the physics determining the pedestal width in order to make first principles calculations of the pedestal height.

In the peeling-ballooning picture $[2,3]$, the sharp pressure gradients and consequent large bootstrap current in the pedestal region provide the free energy which can destabilize peeling (i.e., edge localized external kink) and ballooning modes over a wide range of toroidal mode 
numbers $(n)$. The bootstrap current plays a complex dual role in the stability physics, on one hand driving peeling modes, while on the other lowering edge shear and opening second stability access to high- $n$ ballooning modes. Field line bending stabilizes long wavelength modes, while short wavelengths are stabilized by a combination of second stability and FLR/diamagnetic effects, shifting the limiting modes to intermediate wavelengths. These dominant modes are referred to here as coupled "peeling-ballooning" modes, and are driven by both parallel current $\left(\mathrm{J}_{\text {ped }}\right)$ and the pressure gradient $\left(\mathrm{p}_{\text {ped }}^{\prime}\right)$. These intermediate- $n$ peeling-ballooning modes impose constraints on the pedestal height, which are functions of the pedestal width, plasma shape, collisionality, safety factor and other equilibrium details. Figure 1 provides a schematic rendering of these stability bounds and how they vary with shape. Note that the current in the edge is generally primarily driven by the bootstrap effect, and is therefore roughly proportional to the pressure gradient in steady state, but reduced by collisionality. Because of this collisionality dependence, discharges at very high density will have low values of the current at a given $\mathrm{p}_{\text {ped }}^{\prime}$ and will therefore encounter the "ballooning" instability boundary at the lower right as $\mathrm{p}_{\text {ped }}^{\prime}$ increases, while discharges at very low density will have nearly the full collisionless bootstrap value of $\mathrm{J}_{\text {ped }}$ and may encounter the "peeling" instability boundary at the top of Fig. 1. In the usual intermediate range of densities the instability boundary will be encountered at the upper right where intermediate n peeling-ballooning modes are limiting. These different stability boundaries are associated with modes of varying characteristics which may be associated with different types of ELM cycle as discussed in Refs. 2 and 3. We emphasize that the calculations presented in this paper are for ideal MHD instabilities, and that these instabilities are expected to provide pedestal constraints which pertain for high performance, high power H-mode plasmas (with so-called "Type I" and "Type II" ELMs). There also exist low power regimes, such as the high collisionality variant of "Type III" ELMs, which appear to be constrained, perhaps by resistive MHD physics, to values below the ideal constraints calculated here. However, the high power, lower collisionality regimes are expected to be more relevant both for optimizing performance of current devices, and for Next Step fusion devices. 


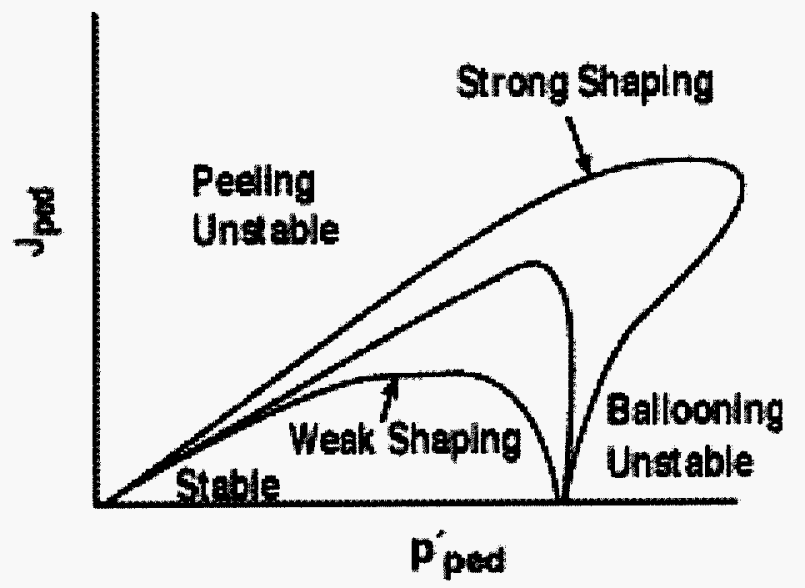

Fig. 1 Schematic diagram of peeling-ballooning stability boundaries as a function of pedestal pressure gradient and current. Boundaries for weak, intermediate and strong shaped discharges are characterized.

A number of studies have compared observed ELM and pedestal behavior to calculated stability bounds using experimentally reconstructed equilibria [3-8]. These studies have generally found that the observation of Type I ELMs can be quantitatively associated with the crossing of MHD stability boundaries, and that observed characteristics of ELMs are qualitatively consistent with calculated unstable mode structures and hybrid growth times. As an example, we show in Fig. 2 calculated mode structures for low (a) and high (b) density DIII-D discharges described in Ref. 8. Both discharges are found to be peeling-ballooning unstable just before the ELM is observed, with broad mode structures calculated for the low density, large ELM case, and narrow mode structures for the high density small ELM case (note that issues of ELM dynamics and ELM size are beyond the scope of this paper, but have been addressed qualitatively in the literature $[2,3,5-10]$ and will be the subject of future studies). 

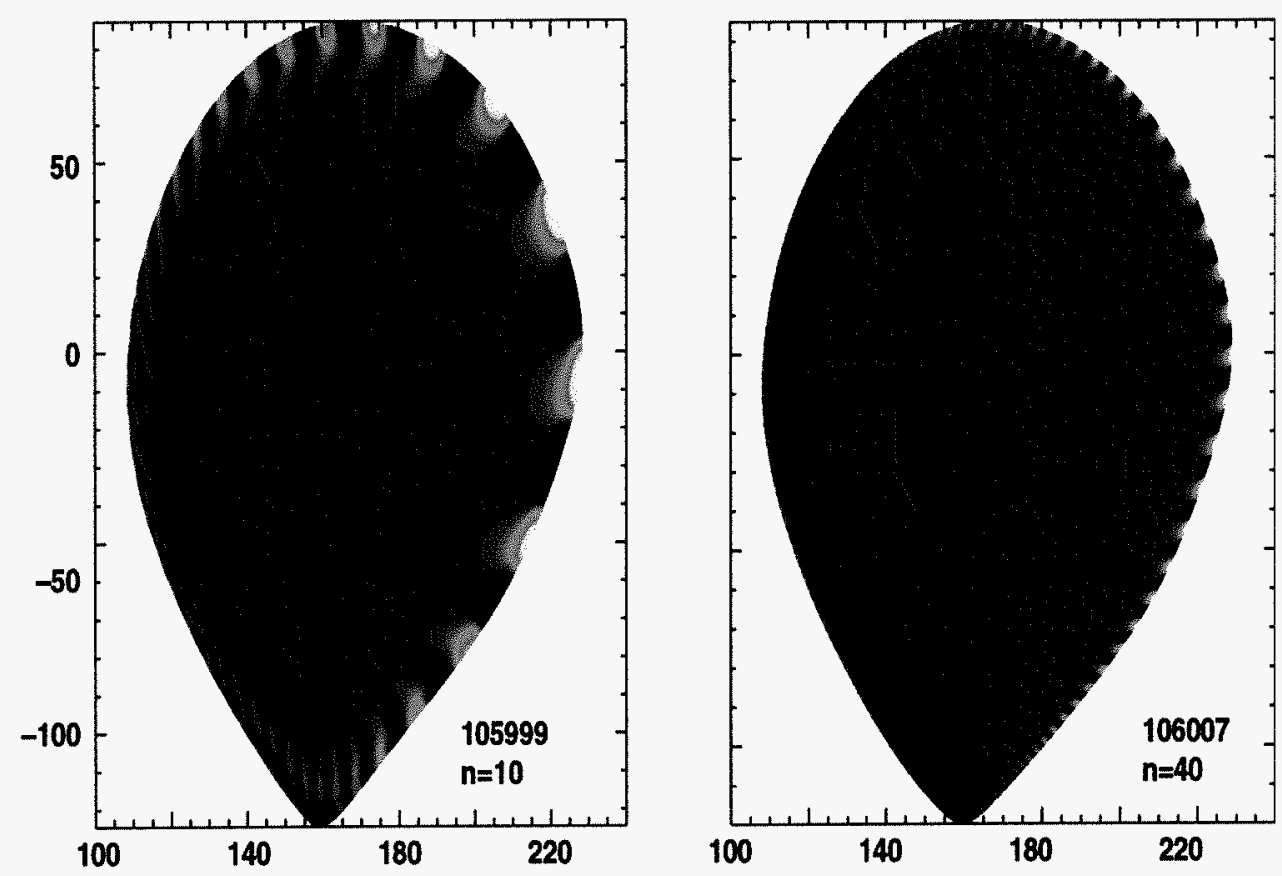

Fig. 2 Unstable mode structures calculated by ELITE just before an ELM for (a) low density plasma with large ELMs (b) high density plasma with small ELMs. 


\section{CHARACTERIZING PEELING-BALLOONING STABILITY BOUNDS}

Direct stability calculations on experimental equilibria allow interpretation of observed edge behavior and provide a useful check of the validity of the peeling-ballooning model. However, because they require detailed profile information from experiment, they do not provide a method to extrapolate or predict pedestal constraints and ELM behavior in future experiments. For this purpose, we employ a technique using model equilibria which are characterized by a relatively small number of important parameters. We follow the technique outlined in Refs. 9 and 10.

\subsection{Technique for Equilibrium Construction and Stability Boundary Calculation}

A general solution to the Grad-Shafranov equation for 2D ideal MHD equilibrium allows two free profile functions as well as a free boundary shape, and thus, in general, an infinite number of OD parameters are required to fully specify the equilibrium. To conduct a practical stability study, it is necessary to limit the number of these parameters, for example by choosing simple functional forms for the profile functions and boundary shape. Such stability studies using parameterized equilibria have been carried out, both for low $\mathbf{n}$ kink modes and for infinite $\mathbf{n}$ ballooning modes, for example in the well known Troyon beta limit study. Here we follow a related approach to study intermediate $n$ peeling-ballooning constraints on the pedestal. We employ profile functions optimized to take advantage of known pedestal characteristics, such as approximately hyperbolic tangent shape of profiles and large bootstrap current in the edge barrier region, and follow a procedure designed for the calculation of pedestal stability constraints as a function of a relatively small number of key parameters. These key parameters are taken to be the pedestal width $(\Delta)$, the toroidal magnetic field $\left(B_{t}\right)$, total plasma current $\left(I_{p}\right)$, major radius $(R)$, minor radius (a), pedestal electron density ( $\left.n_{\text {eped }}\right)$, plasma elongation ( $\left.\kappa\right)$, and triangularity $(\delta)$. Density and temperature profiles are given a hyperbolic tangent shape in the pedestal 
(resembling measured profiles), and a simple polynomial dependence in the core (where details of the profile shape impact pedestal stability less strongly):

$$
\begin{aligned}
\mathrm{n}_{\mathrm{e}}(\psi) & =\mathrm{n}_{\text {sep }}+\mathrm{a}_{\mathrm{n} 0}\left\{\tanh \left[2\left(1-\Psi_{\text {mid }}\right) / \Delta\right]-\tanh \left[2\left(\Psi-\Psi_{\text {mid }}\right) / \Delta\right]\right\} \\
& +\mathrm{a}_{\mathrm{n} 1} \mathrm{H}\left[1-\left(\Psi / \Psi_{\text {ped }}\right)^{\alpha_{\mathrm{n} 1}}\right]^{\alpha_{\mathrm{n} 2}} \\
\mathrm{~T}(\psi) & =\mathrm{T}_{\text {sep }}+\mathrm{a}_{\mathrm{T} 0}\left\{\tanh \left[2\left(1-\Psi_{\text {mid }}\right) / \Delta\right]-\tanh \left[2\left(\Psi-\Psi_{\text {mid }}\right) / \Delta\right]\right\} \\
& +\mathrm{a}_{\mathrm{T} 1} \mathrm{H}\left(1-\Psi / \Psi_{\text {ped }}\right)\left[1-\left(\Psi / \Psi_{\text {ped }}\right)^{\alpha_{\mathrm{r} 1}}\right]^{\alpha_{\mathrm{r} 2}}
\end{aligned}
$$

where $\Psi$ is the normalized poloidal flux, $\Delta$ is the pedestal width in $\Psi$ space, and $H$ is the Heaviside step function. In addition to the key parameters mentioned above, a number of other parameters must be specified to uniquely determine the equilibrium. These include the separatrix values of density and temperature $\left(\mathrm{n}_{\text {sep }}\right.$ and $\left.\mathrm{T}_{\text {sep }}\right)$, the radial location of the steep gradient pedestal region (given by $\Psi_{\text {mid }}$ and $\Psi_{\text {ped }}=\Psi_{\text {mid }}-\Delta / 2$ ), the core profile form parameters $\alpha_{1}$ and $\alpha_{2}$, and the axis values $\mathrm{T}_{0}$ and $\mathrm{n}_{0}$, which together with the pedestal values determine the $a_{0}$ and $a_{1}$ coefficients above. In the pedestal region, the parallel current is taken to be equal to the bootstrap current as calculated using the Sauter collisional model [11] [note that no additional fast equilibrium variation, orbit squeezing, or boundary effects are added to the Sauter model, and that the ion mass $\left(m_{i}\right)$ and average charge state $\left(Z_{\text {eff }}\right)$ or its profile must be specified, and that Ohmic current, if present, is taken to be small compared to the bootstrap current in the sharp gradient pedestal region]. In the core, where details of the current are relatively unimportant, the profile is taken to have a simple polynomial form, with coefficients chosen to give a specified central $\mathrm{q}_{0}$ and the desired $\mathrm{I}_{\mathrm{p}}$. A number of simplifications are made to streamline the equilibrium construction process, including up-down symmetry (while matching the given separatrix elongation and triangularity), and lack of true $\mathrm{X}$-points. Note that the widths of the temperature and density pedestals are taken to be equal in this study for simplicity, but that the effects of differing widths for $\mathrm{T}$ and $\mathrm{n}_{\mathrm{e}}$ can be taken into account in a similar study simply by defining separate $\Delta_{\mathrm{h}}$ and $\Delta_{\mathrm{T}}$ in Eqs 1 and $1 \mathrm{a}$ above. 
For a given set of the above equilibrium parameters, the pedestal temperature is then increased until MHD stability boundaries are crossed. Here we employ the ELITE code [12,3], a highly efficient 2D MHD code designed for the study of edge localized modes, to test stability to a range of modes, $\mathrm{n}=6,8,10,15,20,30$. A standard set of physics assumptions is employed for these calculations of instability thresholds for ideal, external MHD modes. The plasma in the closed flux region is taken to be ideally conducting, and to be surrounded by a vacuum region which models the relatively cold, resistive plasma on open field lines.

In this way, we calculate the maximum stable pedestal temperature as a function of the given equilibrium parameters, $\mathrm{T}_{\text {pedmax }}=\varkappa \Delta, \mathrm{B}_{\mathrm{t}}, \mathrm{I}_{\mathrm{p}}, \mathrm{R}, \mathrm{a}, \kappa, \delta ; \mathrm{n}_{\text {sep }}, \mathrm{T}_{\text {sep }}, \Psi_{\text {mid }}, \alpha_{1}, \alpha_{2}, \mathrm{~T}_{0}, \mathrm{n}_{0}, \mathrm{q}_{0}, \mathrm{~m}_{\mathrm{i}}, \mathrm{Z}_{\mathrm{eff}}$, where the parameters in front of the semicolon are taken to be the "key" parameters whose dependencies will be the focus of this study. Note that both sides of the above equation can of course be multiplied by $n_{\text {ped }}$ and other appropriate factors to give the stability limit in terms of $\mathrm{p}_{\text {pedmax }}, \beta_{\text {pedmax }}, \beta_{\text {Npedmax }}$ etc.

We note that the stability bound $f$ above can, with sufficient calculation, be characterized over a broad range of parameters. This calculation does not in principle require any reference to experiment, and can be performed for parameters in the range of present experiments as well as for those expected in future experiments. Of course to compare to observations one must calculate $f$ in the range of parameters spanned by existing experimental pedestal databases. This is undertaken in the following subsection 2.2. Then in subsection 2.3, parameter scans of the "key" parameters are performed to study their dependencies over a broad range.

\subsection{Comparison to Observation}

The ability of the above technique to accurately calculate pedestal height constraints (as a function of the pedestal width, which is taken as an input parameter here) will depend both on the correctness of the peeling-ballooning model, and on the extent to which the model equilibria provide sufficiently accurate representations of the true experimental equilibria. This can be tested by choosing sets of equilibrium parameters for which experimental data is available, 
calculating stability bounds using model equilibria, and then comparing them to observed pedestal heights in discharges with approximately the chosen key equilibrium parameters. Here we review three such comparisons using parameters and data from the DIII-D tokamak [10]. In these calculations the non-"key" parameters are given values approximately in the observed range; here $\mathrm{n}_{0}=1.5 \mathrm{n}_{\text {eped }}, \mathrm{n}_{\text {sep }}=0.25 \mathrm{n}_{\text {eped }}, \mathrm{T}_{\text {sep }}=100 \mathrm{eV}, \alpha_{\mathrm{n} 0}=1, \alpha_{\mathrm{n} 1}=1.1, \alpha_{\mathrm{T} 0}=1$, $\alpha_{\mathrm{T} 0}=2, \Psi_{\mathrm{mid}}=1-\Delta / 2, \mathrm{q}_{0}=1.05, \mathrm{~m}_{\mathrm{i}}=2, \mathrm{Z}_{\mathrm{eff}}=2$.

The first comparison, shown in Fig. 3(a), studies pedestal height as a function of density, with the axis pressure held fixed $\left(\mathrm{n}_{0} \mathrm{~T}_{0}=210^{20} \mathrm{keV} \mathrm{m} \mathrm{m}^{-3}\right)$, using the parameters $B_{t}=2 \mathrm{~T}$, $\mathrm{I}_{\mathrm{p}}=1.225 \mathrm{MA}, \mathrm{R}=1.685 \mathrm{~m}, \mathrm{a}=0.603 \mathrm{~m}, \mathrm{~K}=1.77, \delta=0.0$, pedestal width on the outboard midplane of $1.7 \mathrm{~cm}$, and $\mathrm{n}_{\text {eped }}$ varied from 2 to $9 \times 10^{19} \mathrm{~m}^{-3}$. The calculated pedestal stability bound is given by a solid line in Fig. 3(a). We emphasize that all the key parameters except the one studied $\left(\mathrm{n}_{\text {eped }}\right)$ are held fixed in the stability calculation. To compare to the observational pedestal dataset one must of course allow a narrow range of these "fixed" parameters because no points in the dataset will match them all precisely. This range is chosen to be wide enough to include sufficient data to allow a meaningful comparison, yet narrow enough such that variation of these parameters does not unduly skew the comparison. The data is taken from the DIII-D pedestal database, from the last $20 \%$ of the Type I ELM cycle, here with allowed ranges for the "fixed" parameters of $\mathrm{B}_{\mathrm{t}}=1.9-2.05 \mathrm{~T}, \mathrm{I}_{\mathrm{p}}=1.15-1.25 \mathrm{MA}, 0<\delta<0.2$, temperature and density pedestal widths between 1.2 and $2.2 \mathrm{~cm}$, and injected power $P_{\text {inj }}>1 \mathrm{MW}$.

A second comparison studies pedestal height trends with triangularity, using the parameters $B_{t}=2.08 T, I_{p}=1.525 \mathrm{MA}, \kappa=1.8, n_{\text {eped }}=4 \times 10^{19} \mathrm{~m}^{-3}$, temperature and density pedestal width of $1.4 \mathrm{~cm}$, and triangularity varied from 0 to 0.45 . Here the axis temperature is fixed $\left(\mathrm{T}_{0}=2.5 \mathrm{keV}\right)$, and again, in calculating the stability bounds, all of the key parameters are fixed except the one studied $(\delta)$. The result is again compared to DIII-D data, here with allowed parameter ranges $B_{t}=2.05$ to $2.15 T, I_{p}=1.4$ to $1.65 \mathrm{MA}, n_{\text {eped }}=3.5$ to $4.5 \times 10^{19} \mathrm{~m}^{-3}$, and temperature and density pedestal widths between 0.9 and $1.9 \mathrm{~cm}$. Good agreement is again found between the calculated pedestal stability bound and the observed pedestal height shortly 
before an ELM as shown in Fig. 3(b). The increase in the stability bound with triangularity is primarily due to an opening of second stability access, and the bootstrap current plays a key role as shown schematically in the "strong shaping" curve in Fig. 1. Without the bootstrap current [dashed line in Fig. 3(b)], second stability access is not opened and the increase in stable pedestal height with triangularity is much weaker.
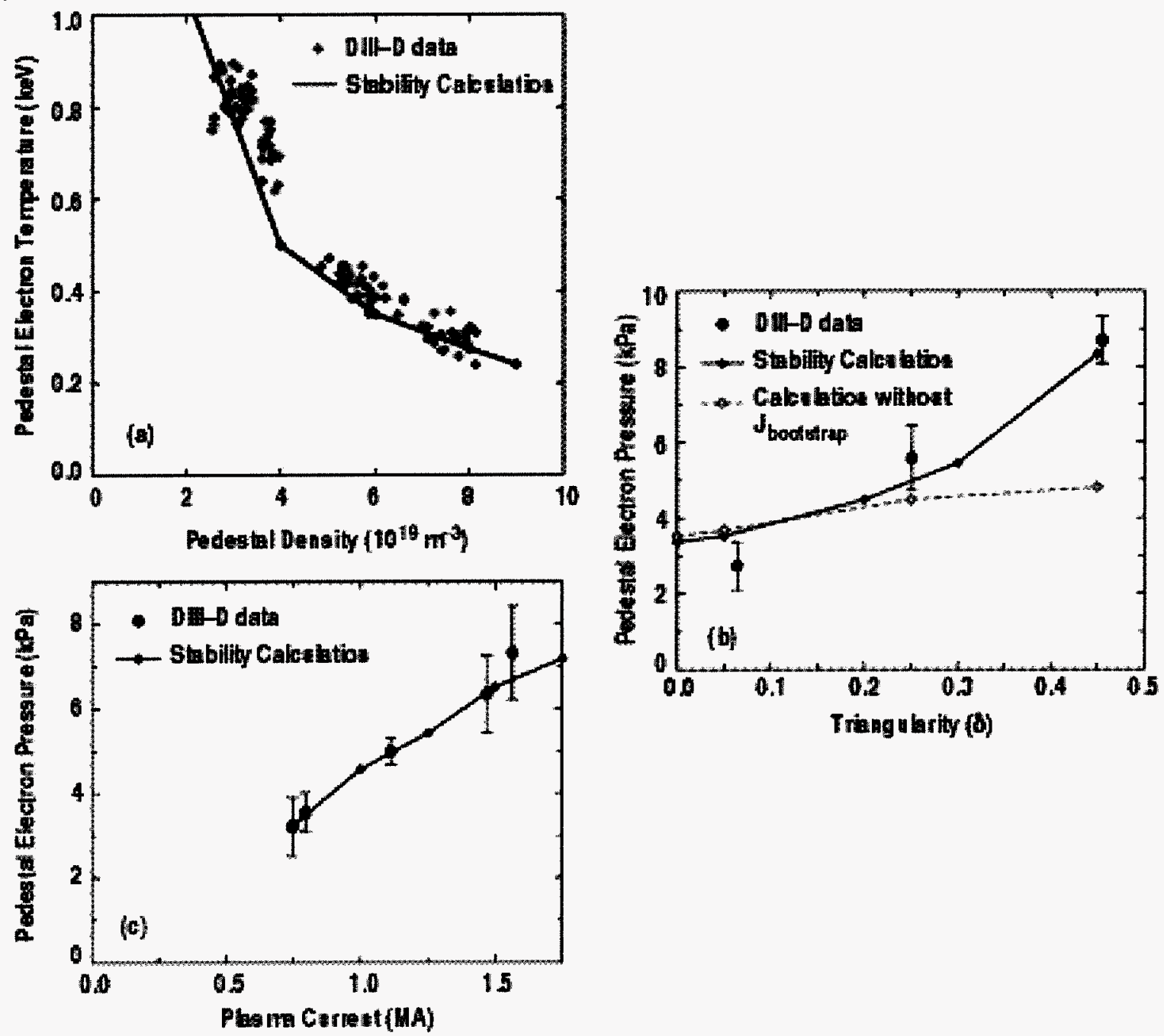

Fig. 3 Comparison of pedestal stability limit calculated with ELITE to DIII-D data as a function of (a) pedestal density (b) triangularity and c) plasma current.

Finally, we study trends in pedestal height with $I_{p}$, using equilibria with $B_{t}=2.075 \mathrm{~T}, \mathrm{R}=$ $1.69 \mathrm{~m}, \mathrm{a}=0.59 \mathrm{~m}, \kappa=1.8, \delta=0.25$, pedestal width $(\Delta)$ of $4.5 \%$ of the normalized poloidal flux, and pedestal density ( $\left.\mathrm{n}_{\text {eped }}\right) 40 \%$ of the Greenwald limit $\left(\mathrm{n}_{\mathrm{GW}}\right)$, where $\mathrm{n}_{\mathrm{GW}}\left(10^{20} \mathrm{~m}^{-3}\right)=$ $I_{p}(M A) / \pi a^{2}(m)$. The current $\left(I_{p}\right)$ is varied from 0.75 to $1.75 \mathrm{MA}$, with core temperature 
$\left(\mathrm{T}_{0}=2.975 \mathrm{eV}\right)$, and thus core $\beta_{\mathrm{N}}$, fixed. Here ELITE is used to test stability for $5>\mathrm{n}>30$, and the DCON code is used to confirm stability at low $n$, giving the stability boundary shown in Fig. 3(c). The stability bound is again compared to DIII-D pedestal data in the final $20 \%$ of the ELM cycle, with parameter ranges $2.05 \mathrm{~T}<\mathrm{B}_{\mathrm{t}}<2.1 \mathrm{~T}, 0.3<\mathrm{n}_{\text {eped }} / \mathrm{n}_{\mathrm{GW}}<0.5,0.2<\delta<0.4$, and $3.5 \%<\Delta<5.5 \%$.

The level of agreement in all three studies shown in Fig. 3 is strongly encouraging. Note that there are no adjustable parameters in the stability calculations, and that both the absolute values and the trends with density, triangularity and current are reproduced with reasonable accuracy. This agreement strongly encourages the use of this technique to predict pedestal behavior in future experiments as has been done for ITER in Ref. 9. However, while most of the key parameters needed to construct the model equilibria and calculate stability bounds (e.g., $B_{t}, I_{p}$, $\mathrm{n}_{\text {eped }}, \kappa, \delta$ ) are known or expected to be controllable in future experiments, the pedestal width $\Delta$ is not known, and no widely accepted first principles model exists. Hence, pedestal stability bounds are calculated as a function of the width and can be used to study trends in the observed width and to provide a key component of models which seek to explain the physics of the pedestal width.

\subsection{Characterization of functional variations of peeling-ballooning stability boundaries}

Here we provide an extensive set of calculated peeling-ballooning stability boundaries, which elucidate the underlying stability physics and should provide useful input for modelers attempting to characterize pedestal stability bounds more accurately (e.g., Refs. 13-15).

We carry out a series of pedestal stability scans, using as a baseline DIII-D-like equilibrium parameters $\mathrm{B}_{\mathrm{t}}=2 \mathrm{~T}, \mathrm{I}_{\mathrm{p}}=1.225 \mathrm{MA}, \mathrm{R}=1.685 \mathrm{~m}, \mathrm{a}=0.603 \mathrm{~m}, \kappa=1.77$, and calculating stability bounds with ELITE for $5<\mathrm{n}<30$ and with DCON for $1 \leq \mathrm{n}<6$ (with a conformal wall at 1.2). Near resonant conditions are disallowed $\left(m_{0}-n q_{a}>0.1\right)$. The non-key parameters are given values similar to those in the previous subsection $\left(\mathrm{n}_{0}=1.5 \mathrm{n}_{\text {eped }}, \mathrm{n}_{\text {sep }}=0.25 \mathrm{n}_{\text {eped }}\right.$, $\left.\mathrm{T}_{\mathrm{sep}}=100 \mathrm{eV}, \alpha_{\mathrm{n} 0}=1, \alpha_{\mathrm{n} 1}=1.1, \alpha_{\mathrm{T} 0}=1, \alpha_{\mathrm{T} 0}=2, \Psi_{\mathrm{mid}}=1-\Delta / 2, \mathrm{q}_{0}=1.05, \mathrm{~m}_{\mathrm{i}}=2\right)$, with the 
exception that $Z_{\text {eff }}=2.5$, and $T_{0}$ is specified by a constant core pressure constraint $\left(\mathrm{n}_{0} \mathrm{~T}_{0}=2 \quad 10^{20}\right.$ $\left.\mathrm{keV} \mathrm{m}^{-3}\right)$.

Scans of the maximum stable pedestal height as a function of pedestal width are shown in Fig. 4, for $\delta=0$ and two values of the pedestal density. The maximum stable height increases with pedestal width as expected, but the rate of increase is less than linear; that is the pedestal stability limit is not strictly a limit on the pressure gradient. This is because (1) finite n modes are non-local and are directly sensitive to the pedestal width as well as local parameters, and (2) the natural magnetic shear in the middle of the pedestal where gradients are steepest decreases with pedestal width. This effect is illustrated in plots of normalized pressure gradient $(\alpha)$ and magnetic shear in Fig. 4(b). Fitting the calculated height limit to a functional form $\mathrm{p}_{\text {ped }} \sim \Delta^{\mathrm{c}}$ yields a coefficient $\mathrm{c} \sim 0.7$ for the high density and $c \sim 0.8$ for the low density cases.
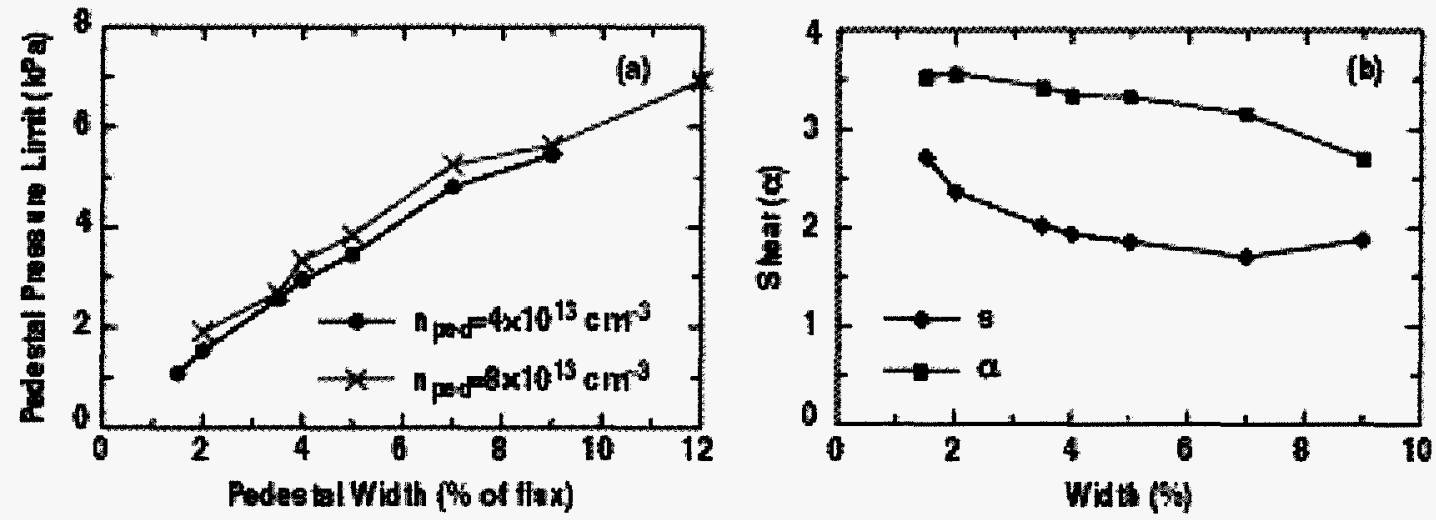

Fig. 4. (a) Calculated pedestal pressure stability limit as a function of pedestal width for two densities, and (b) magnetic shear and normalized pressure gradient as a function of width for the $n_{\text {ped }}=8 \times 10^{13} \mathrm{~cm}^{-3}$ case.

The scaling of the pedestal stability bounds with density can be quite complex as illustrated in Fig. 5. At low triangularity [Fig. 5(a)], there is no second stability access and the pedestal stability limit increases slightly with density due to the reduction in bootstrap current (i.e., increase in magnetic shear) at higher collisionality as in the simple first stable high-n ballooning s-alpha diagram. However, at higher triangularity [Figs. 5(b) and 5(c)] second stability access is opened at intermediate densities. The stability boundary then decreases at high density due to collisional reduction of the bootstrap current which weakens second stability access and also decreases at very low density due to the onset of kink/peeling modes. Note that the shape of this 
curve is consistent with the "strong shaping" curve in Fig. 1 noting that the stability boundary is encountered in the upper right of the diagram and that current decreases with density. The overall dependence with triangularity illustrated in Figs. $5(\mathrm{a})-$ (c) scales roughly as $\sim(1+\delta)^{1.3}$ when averaged over all densities, and as $\sim(1+\delta)^{1.7}$ for intermediate density $\left(4 \times 10^{19} \mathrm{~m}^{-3}\right)$.
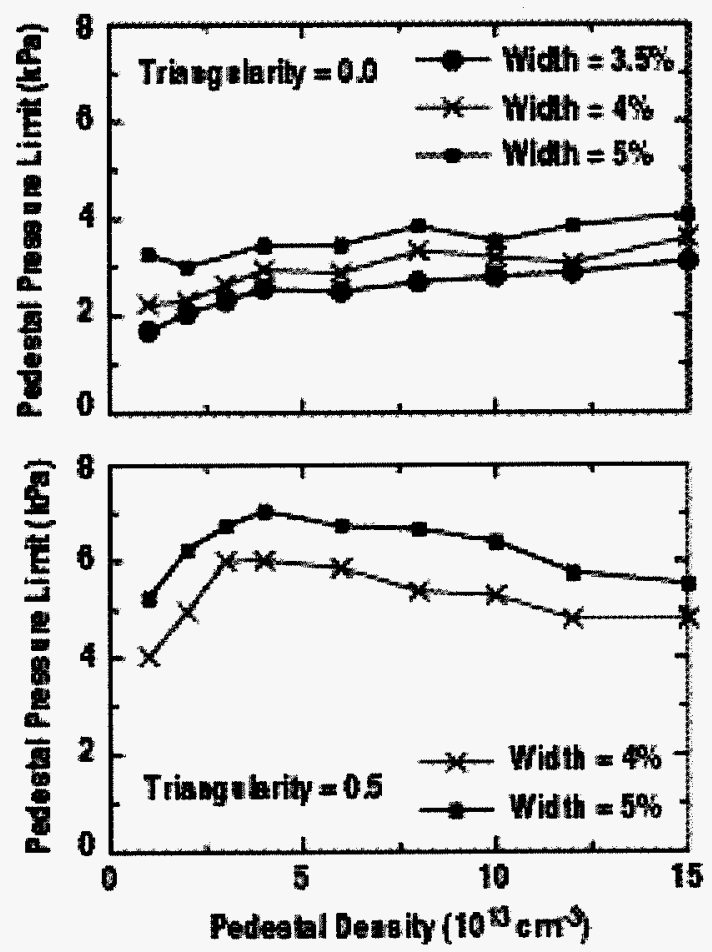

Fig. 5. Maximum stable pedestal pressure as a function of pedestal density for (a) triangularity $=0$ (b) triangularity $=0.3(\mathrm{c})$ triangularity $=0.5$, for a range of pedestal widths.

Scaling of stability bounds with magnetic field and plasma current is shown in Fig. 6 for $\delta=0.0$ and 0.3 , and $\mathrm{n}_{\text {ped }}=8 \times 10^{19} \mathrm{~m}^{-3}$. In Fig. 6(a) the current is increased at constant $B_{t}$, resulting in a decreasing safety factor (q), in Fig. $6(\mathrm{~b}) \mathrm{B}_{\mathrm{t}}$ is increased at constant $I_{p}$, resulting in an increasing $q$, and in Fig. 6(c) $I_{p}$ and $B_{t}$ are both proportionally increased, resulting in an approximately constant $\mathrm{q}$. For the low triangularity case, maximum stable pedestal height increase strongly with $I_{p}\left(\sim I_{p}^{0.9}\right)$, somewhat less strongly with $B_{t}\left(\sim B_{t}^{0.6}\right)$ due to increasing $q$ which increases $\alpha$ at a given pressure gradient and roughly linearly with $B_{t}^{*} I_{p}$ when both are increased proportionally. In the strongly shaped case $(\delta=0.3)$, second stability is closed off by low $\mathrm{q}$ for both high current or low $\mathrm{B}_{\mathrm{t}}$, resulting in the behavior shown in Figs. 6(a) and (b). Again, when $B_{t}$ and $I_{p}$ are proportionally increased, a roughly linear dependence on $B_{t}^{*} I_{p}$ is 
seen. Overall the scaling with $\mathrm{B}_{\mathrm{t}}$ and $\mathrm{I}_{\mathrm{p}}$ is roughly a $\beta_{\mathrm{N}}$ dependence [where $\beta=\beta_{\mathrm{N}}$ $\mathrm{I}(\mathrm{MA}) / \mathrm{a}(\mathrm{m}) \mathrm{B}(\mathrm{T})]$, though complex interdependencies enter, particularly for strong shaping.
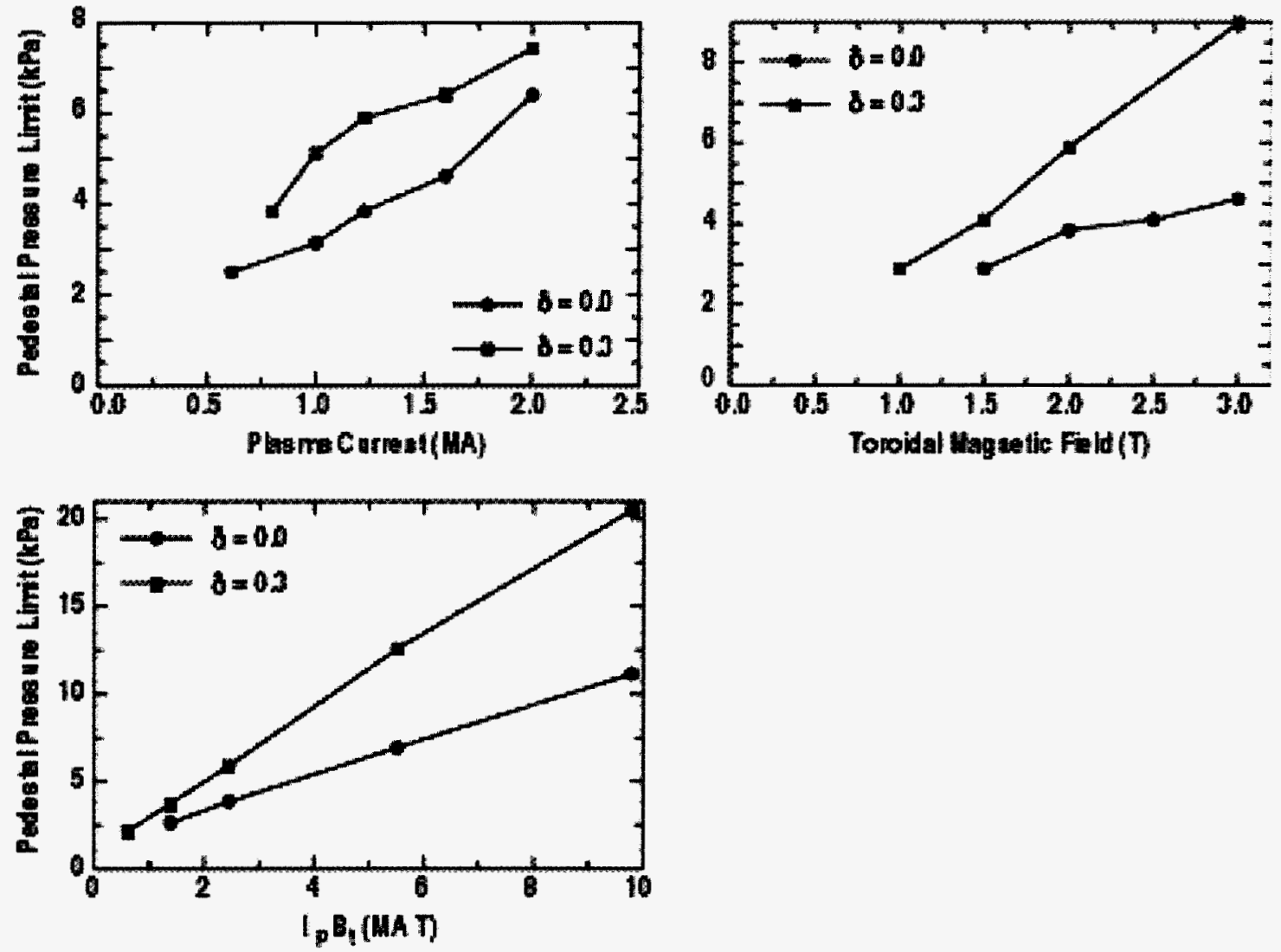

Fig. 6. Maximum stable pedestal pressure for triangularity $=0.0$ and 0.3 cases as a function of (a) plasma current with $B_{t}$ constant (b) magnetic field with constant current, (c) magnetic field and plasma current increases proportionally with ratio $B_{t} / I_{p}=0.6125 \mathrm{~T} / \mathrm{MA}$.

Finally, scaling with aspect ratio is illustrated via a major radius scan at fixed minor radius in Fig. 7. Both $\mathrm{q}$ and $\mathrm{a} / \mathrm{R}$ decrease roughly linearly with $\mathrm{R}$. At low triangularity $(\delta=0)$ the stability boundary is found to be fairly insensitive to aspect ratio. However, for the stronger shaped case $(\delta=0.3$ ), second stability is closed off at high R/a (low q) and the stability boundary approaches the $\delta=0$ bound. 


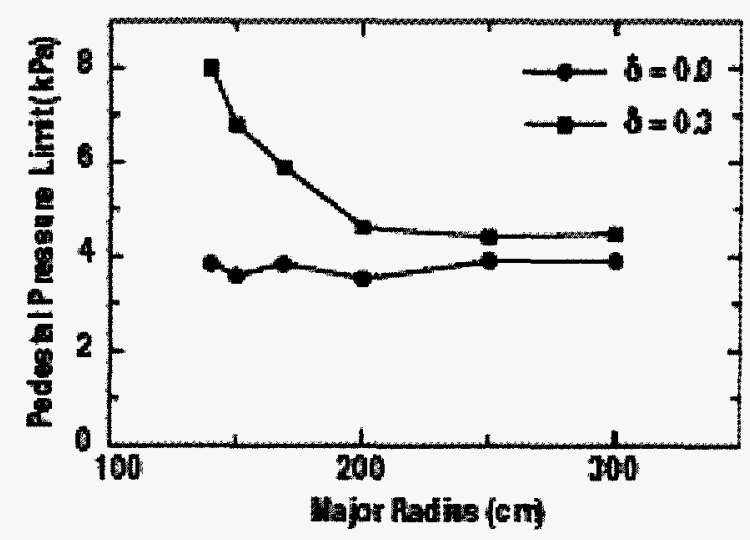

Fig. 7. Maximum stable pedestal pressure for triangularity $=0.0$ and 0.3 cases as a function of aspect ratio, which is varied by changing major radius at a fixed minor radius of $60.3 \mathrm{~cm}$. 


\section{DISCUSSION}

Encouraging agreement between calculated peeling-ballooning stability bounds and observed pedestal constraints has been reported in several studies (e.g., Refs. 3-8). Here we discuss a technique employing model equilibria to calculate pedestal stability boundaries as a function of important equilibrium quantities $\left(\mathrm{B}_{\mathrm{t}}, \mathrm{I}_{\mathrm{p}}, \mathrm{n}_{\text {eped }}, \kappa, \delta, \Delta, \ldots\right)$. This technique allows for comparisons to existing pedestal data as well as predictions for future experiments and calculations of general parameter variation of the pedestal stability constraints. Good agreement is found between calculated stability bounds and observed pedestal height shortly before ELMs on the DIII-D tokamak. The absolute value of the calculated pedestal height constraint as well as trends with density, triangularity and current are reproduced. The pedestal width $(\Delta)$ is taken as an input in these calculations. For parameterization and predictive studies, the maximum stable pedestal height can be calculated as a function of the pedestal width and used either in conjunction with or for the development of pedestal width models.

We conduct an extensive set of studies characterizing the peeling-ballooning stability constraints (calculated for $\mathrm{n}=1$ to 30 with ELITE and DCON) as a function of pedestal width, density, triangularity, current, magnetic field, and aspect ratio. The complex behavior of the peeling-ballooning stability physics, for example the dual role of the bootstrap current in opening second stability access to ballooning modes while also destabilizing peeling modes, makes simple parameterization of the stability bounds difficult. For example, multi-parameter dependencies are introduced by the collisionality dependence of the bootstrap current and the influence of shape and $\mathrm{q}$ on second stability access. However, some approximate conclusions can be drawn. The pedestal height stability limit increases strongly with pedestal width as expected, but it is not simply a pressure gradient limit, instead increasing roughly as $\Delta^{0.8}$ due to profile and finite $\mathrm{n}$ effects. The current and magnetic field dependencies can be approximated as a $\beta_{\mathrm{N}}$ dependence, and recasting pedestal stability limits in terms of $\beta_{\mathrm{Nped}}$ introduces a useful 
metric. We note that for Figs. 4, 5 and 7 the illustrated $\mathrm{p}_{\text {ped }}$ limits can be converted to $\boldsymbol{\beta}_{\mathrm{Nped}}$ limits via the relationship $\beta_{\mathrm{Nped}} \sim 0.13 \mathrm{p}$ ped $(\mathrm{kPa})$. Access to second stability can significantly increase pedestal stability limits, but it requires a somewhat delicate balance of parameters, notably strong shaping, intermediate density, and moderate q. The observed triangularity dependence in this study averaged over all densities is roughly $\sim(1+\delta)^{1.3}$, but scales as $\sim(1+\delta)^{1.7}$ for intermediate density. We note that these scalings should be used with care due to the complex multi-parameter dependencies; and that for projection to future experiments, these rough scalings should be complemented by a detailed pedestal stability study for the relevant parameters.

The pedestal stability calculations discussed here employ a static ideal MHD model. While encouraging agreement has been found between such calculations and experimental observations, it remains important to consider additional physics such as toroidal rotation shear and diamagnetic effects which can modify ideal stability limits. A detailed study of toroidal rotation shear effects using ELITE is underway and will be reported in a future publication. In addition, nonlinear studies of peeling-ballooning mode dynamics using the reduced Braginskii code BOUT [16] are being conducted to evaluate two fluid effects and explore the nonlinear dynamics needed for addressing important questions about ELM amplitude and heat loads to material surfaces. 


\section{ACKNOWLEDGMENTS}

Work supported by U.S. Department of Energy under Contract No. DE-AC0399ER54463 and by the UK Dept. of Trade and Industry and EURATOM. 


\section{REFERENCES}

[1] A.D. Turnbull, M.A. Secretan, F. Troyon, et al., J. Comp. Phys. 66 (1986) 391; J. Manickam, Phys. Fluids B 4 (1992) 1901; G.T.A. Huysmans, C.D. Challis, M. Erba, et al., Proc. EPS Conf. on Control. Fusion and Plasma Physics, 1995.

[2] J.W. Connor, et al., Phys. Plasmas 5 (1998) 2687; C.C. Hegna, et al., Phys. Plasmas 3 (1996) 584.

[3] P.B. Snyder, J.R. Wilson, J.R. Ferron et al., Phys. Plasmas 9 (2002) 2037.

[4] D. Mossessian, P.B. Snyder, M. Greenwald, et al., Plasma Phys. Control. Fusion 44 (2002) 423-437; D.A. Mossessian, P. Snyder, A. Hubbard et al., Phys. Plasmas 10 (2003) 1720.

[5] L.L. Lao, Y. Kamada, T. Okawa, et al., Nucl. Fusion 41 (2001) 295.

[6] J.R. Ferron et al. Phys. Plasmas 7 (2000) 1976.

[7] S. Saarelma, et al., Plasma Phys. Control. Fusion 42 (2000) A139; S. Saarelma, et al., Nucl. Fusion 43 (2003) 262.

[8] A.W. Leonard, et al., Phys. Plasmas 10 (2003) 1765.

[9] P.B. Snyder, H.R. Wilson, Plasma Phys. Control. Fusion 45 (2003) 1671.

[10] P.B. Snyder, H.R. Wilson, J.R. Ferron et al., "ELMs and Constraints on the H-Mode Pedestal: Peeling-Ballooning Stability Calculation and Comparison to Experiment," Proc. 19th IAEA Fusion Energy Conf., October 14-19, 2002, Lyon, France (International Atomic Energy Agency, Vienna, 2002) CD-ROM; submitted to Nucl. Fusion.

[11] O. Sauter, C. Angioni, Y.R. Lin-Liu, Phys. Plasmas 6 (1999) 2834; O. Sauter, C. Angioni, Y.R. Lin-Liu, Phys. Plasmas 9 (2002) 5140.

[12] H.R. Wilson, P.B. Snyder, et al., Phys. Plasmas 9 (2002) 1277.

[13] M. Sugihara, et al., Plasma Phys. Control. Fusion 45 (2003) L55. 
[14] G. Janeschitz, et al., Plasma Phys. Control. Fusion 44 (2002) A459; G. Bateman et al., Plasma Phys. Control. Fusion 45 (2003) 1939.

[15] J.-S. Lonnroth et al., Plasma Phys. Control. Fusion 45 (2003) 1869.

[16] X.Q. Xu, et al., New J. Physics 4 (2002) 53. 